Research Article:

\title{
Preoperative Clinical Correctability and Prediction of the Prosthesis Type in Total Knee Arthroplasty for Severe Os- teoarthritic Varus Deformity
}

Omid Shahpari $^{1}$ (D), Abolfazl Bagherifard ${ }^{1}$ (D), Mahmoud Jabalameli ${ }^{1}$ (D), Mohammad Rahbar ${ }^{1}$ (D), Hossein Hajitaghi ${ }^{\text {* }}$ (D)

1. Bone and Joint Reconstruction Research Center, Shafa Orthopedic Hospital, Iran University of Medical Sciences, Tehran, Iran.

Crtation Shahpari O, Bagherifard A, Jabalameli M, Rahbar M, Hajitaghi H. Preoperative Clinical Correctability and Prediction of the Prosthesis Type in Total Knee Arthroplasty for Severe Osteoarthritic Varus Deformity. Journal of Research in Orthopedic Science. 2019; 6(4):1-6. http://dx.doi.org/10.32598/JROSJ.6.4.43

oij: : http://dx.doi.org/10.32598/JROSJ.6.4.43

Keywords:

Severe genu varum deformity, Total knee arthroplasty, Constrained condylar knee prosthesis

\begin{abstract}
A B S T R A C T
Background: The preoperative identification of patients who might need Constrained Condylar Knee (CCK) prosthesis in Total Knee Arthroplasty (TKA), is essential to ensure the availability of equipment and to address the patients' expectations accurately.

Objectives: In this study, we aimed at investigating if the preoperative features of the patients can provide this data.

Methods: A total of 30 patients who underwent primary TKA for severe osteoarthritic genu varum deformity (varus angle $\geq 20^{\circ}$ ) were evaluated in this retrospective study. Prosthesis selection was based on preoperative and intraoperative information. Demographic data, preoperative correctability of the deformity, and intraoperative information, including the reduction osteotomy, soft-tissue release, and pie-crust technique, were retrospectively collected. Soft-tissue release was performed in a sequential manner in 3 steps.

Results: The study population included 4 males and 26 females with a Mean \pm SD age of $64.6 \pm 8.7$ years. A CCK prosthesis was used in 11 (36.7\%) cases. A significant association was found between the preoperative correctability and the type of prosthesis. In other words, all CCK prostheses were used in patients who were preoperatively non-correctable $(\mathrm{P}<0.001)$. Also, the step of release was significantly associated with the type of prosthesis, and CCK prosthesis was used in all patients with step 3 release $(\mathrm{P}<0.001)$. Preoperative correctability was significantly related to the step of release, as well. It means that all deformities with step 3 release were preoperatively non-correctable $(\mathrm{P}=0.008)$.
\end{abstract}

Conclusion: The preoperative clinical evaluation of correctability could be used in the identification of patients who might need a CCK prosthesis.

\footnotetext{
* Corresponding Author:

Hossein Hajitaghi, MD.

Address: Bone and Joint Reconstruction Research Center, Shafa Orthopedic Hospital, Iran University of Medical Sciences, Tehran, Iran.

Phone: +98 (21) 33542000-8

E-mail: hosseinhajitaghihh@gmail.com
} 


\section{Introduction}

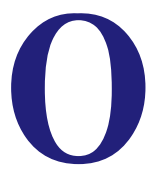

steoarthritis (OA) is one of the leading causes of pain and disability among elderly individuals [1]. The prevalence of knee OA has been reported by $19.34 \%$ in rural areas of Iran in 2014 [2]. As the number of patients over 60 years is estimated to double by $2050, \mathrm{OA}$ is expected to turn into the greatest cause of disability in the general population by $2030[3,4]$.

Total Knee Arthroplasty (TKA) is considered a successful procedure for symptomatic OA, and its number is also expected to increase parallel to the population aging [5]. Nevertheless, up to $30 \%$ of patients are not satisfied with the outcome of TKA [6]. Therefore, improvement of the quality of life of the patients by optimizing TKA results is a healthcare necessity.

A successful TKA aims at achieving optimum alignment, appropriate balance, and deformity correction. In primary TKA, these goals can be attained efficiently by Posterior Stabilized (PS) TKA. However, certain circumstances are extremely difficult to balance and require constrained prostheses even in primary TKA. In such situations, a Constrained Condylar Knee (CCK) design is the prosthesis of choice [7].

Recent investigations reveal that using CCK prosthesis in primary TKA would not be detrimental to the patient and provides similar outcomes compared with PS prosthesis [7, 8]. Even so, it is generally avoided because of its higher cost, the complexity of the procedure, and greater bone removal [9]. Therefore, the implications of CCK prosthesis should be restricted to the patients who have indications.

Preoperative planning is of critical importance in primary TKA and gives the surgeon an insight to anticipate the potential difficulties, as well as to minimize the risk of premature implant failure [10]. The preoperative identification of prosthesis type is also essential as it ensures the availability of appropriate prosthesis and addresses the patients' expectations through accurate preoperative counseling.

To date, prosthesis type is mainly determined based on the intraoperative information. We hypothesized that the preoperative characteristics of the patients, such as clinical correctability, could be used in the preoperative determination of prosthesis type. In this study, in a series of patients with severe osteoarthritic genu varum deformity, we aim at investigating factors that could help in the identification of patients who might require a CCK prosthesis.

\section{Methods}

This retrospective study was approved by the institutional review board of our institute, and written informed consent was obtained from patients to use their medical data. In a retrospective study, patients who underwent primary TKA between 2017 and 2019 for severe osteoarthritic genu varum deformity (varus angle $\geq 20^{\circ}$ ) were evaluated for the eligibility criteria (Figure 1). Patients with the diaphyseal femoral or tibial canal owing to prior trauma, operation, or retained hardware, were excluded. Patients with incomplete medical records were excluded, as well.

A total of 30 patients were eligible to include in the study. All the patients had tibia vara. No patient had recurvatum. All operations were performed under general anesthesia, using a standard medial parapatellar approach. A tourniquet was inflated at the beginning of the procedure and kept inflated until the wound closure. The decision to use a CCK prosthesis was mainly based on the preoperative level of ligamentous stability, intraoperative evaluation of competency of the collateral ligaments, and on-table evaluation of coronal plane stability following the soft-tissue release. In both groups, Zimmer NexGen ${ }^{\circledR}$ Legacy® knee prosthesis was implanted in the majority of cases.

The demographic data were extracted from the patients' medical records. The correctability of the deformity was determined before the operation, and categorized as correctable and non-correctable. In this respect, the knee would be considered correctable if it could clinically approximate the knee alignment to the normal alignment, using a valgus force.

Intraoperative information included the data regarding the performance of reduction osteotomy, soft-tissue release, and the pie-crust technique. Soft-tissue release was performed sequentially and the following steps: Step 1. osteophyte removal and release of the deep medial collateral ligament; Step 2. posterior oblique ligament, and semimembranosus release; Step 3. superficial medial collateral ligament release; and Step 4. pes anserinus tendon release [11].

\section{Statistical analysis}

SPSS V. 16 was used to analyzing the obtained data. The descriptive data were provided as number and 
percentage of Mean \pm SD. The association between the choice of prosthesis and pre- or intra-operative parameters was analyzed using the Chi-square test. A comparison of means between the two study groups was made using the Mann-Whitney $\mathrm{U}$ test. The $\mathrm{P}<0.05$ was considered as significance level.

\section{Results}

A total of 30 patients with a Mean \pm SD age of $64.6 \pm 8.7$ years were included in this study. The study population consisted of $4(13.3 \%)$ males and $26(86.7 \%)$ females. Lateral laxity was present in $7(23.3 \%)$ patients. A CCK prosthesis was used in $11(36.7 \%)$ cases. Fourteen (46.7\%) deformities were preoperatively correctable. The release was performed at step 1 in $3(10 \%)$ patients, at step 2 in $19(63.3 \%)$, and at step 3 in 8 (26.7\%) patients. No step 4 release was performed in this series. Reduction osteotomy was performed in $18(60 \%)$ patients. Pie-crusting was done in $10(33.3 \%)$ patients. Table 1 presents the demographic, preoperative, and intraoperative characteristics of the patients.

Age and sex of the patients were not statistically associated with the type of prosthesis $(\mathrm{P}=0.52$ and $\mathrm{P}=0.73$, respectively). The presence or absence of preoperative lateral laxity was not related to the kind of prosthesis, as well $(\mathrm{P}=0.48)$. A significant association was found between the preoperative correctability of deformities and the type of prosthesis; all CCK prostheses were used in patients who were preoperatively non-correctable $(\mathrm{P}<0.001)$. Also, the step of release was significantly associated with the type of prosthesis so that CCK prosthesis was used in all patients with step 3 release and no patients with step 1 release $(\mathrm{P}<0.001)$. Reduction osteotomy and pie-crusting were not associated with the type of prosthesis $(\mathrm{P}=0.53$ and $\mathrm{P}=0.17$, respectively). Table 2 presents the comparison of the demographic, preoperative, and intraoperative characteristics of the patients.

Preoperative correctability was significantly associated with the step of release; all deformities with step 3 release were preoperatively non-correctable $(\mathrm{P}=0.008)$. No significant association was found between the preoperative correctability and other intraoperative characteristics, as well as demographic features.

\section{Discussion}

Several surgical treatments have been developed for the treatment of osteoarthritic genu varum deformity ranging from attempted osteotomy techniques to TKA $[12,13]$. Severe osteoarthritic genu varum deformities are generally managed with TKA. However, optimizing ligamentous balance and maximizing the range of motion is challenging in these patients and many patients will require a CCK prosthesis. The identification of patients who may need a CCK prosthesis could help the

Table 1. The demographic, preoperative, and intraoperative features of the patients [Mean \pm SD of Age (y): 64.6 \pm 8.7 ]

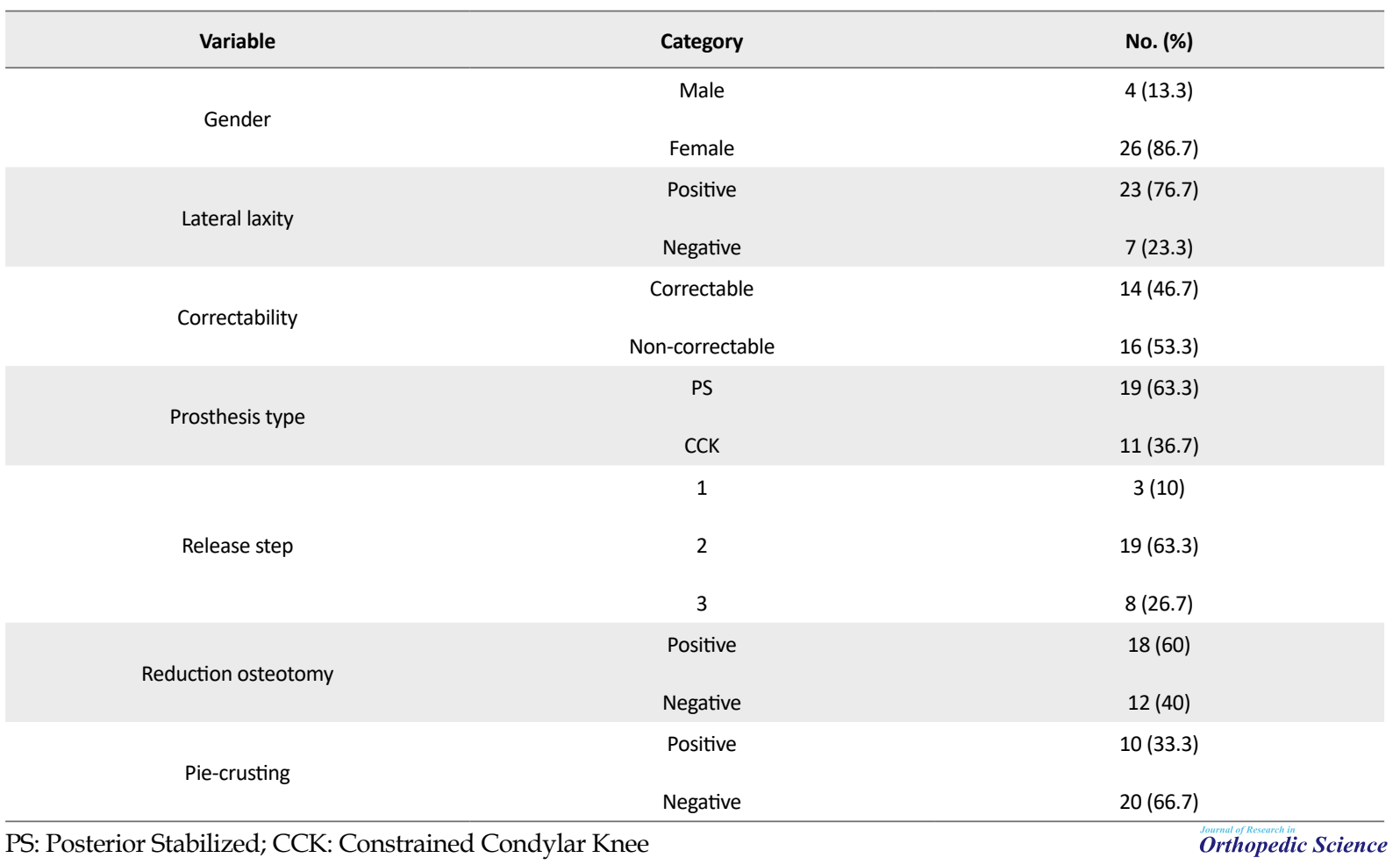


Table 2. Comparison of the demographic, preoperative, and intraoperative characteristics of PS and CCK group of patients

\begin{tabular}{|c|c|c|c|c|}
\hline \multirow{2}{*}{\multicolumn{2}{|c|}{ Variable }} & \multicolumn{2}{|c|}{ Mean \pm SD } & \multirow{2}{*}{ Sig. } \\
\hline & & \multirow{2}{*}{$\begin{array}{c}\text { PS Group ( } n=19 \text { ) } \\
64.2 \pm 8.5\end{array}$} & CCK Group (n=11) & \\
\hline \multicolumn{2}{|c|}{ Age $(y)$} & & $65.3 \pm 8.9$ & 0.52 \\
\hline \multirow{2}{*}{\multicolumn{2}{|c|}{ Variable }} & \multicolumn{2}{|c|}{ No. (\%) } & \multirow{2}{*}{ Sig. } \\
\hline & & PS Group (n=19) & CCK Group (n=11) & \\
\hline \multirow{2}{*}{ Gender } & Male & $3(15.8)$ & $1(9)$ & \multirow{2}{*}{0.73} \\
\hline & Female & $16(84.2)$ & $10(91)$ & \\
\hline \multirow{2}{*}{ Lateral laxity } & Positive & $14(73.7)$ & $9(81.8)$ & \multirow{2}{*}{0.48} \\
\hline & Negative & $5(26.3)$ & $2(18.2)$ & \\
\hline \multirow{2}{*}{ Correctability } & Correctable & $14(73.7)$ & $0(00)$ & \multirow{2}{*}{$<0.001$} \\
\hline & Non-correctable & $5(26.3)$ & $11(100)$ & \\
\hline \multirow{3}{*}{ Release stage } & 1 & $3(15.8)$ & $0(00)$ & \multirow{3}{*}{$<0.001$} \\
\hline & 2 & $16(84.2)$ & $3(27.3)$ & \\
\hline & 3 & $0(00)$ & $8(72.7)$ & \\
\hline \multirow{2}{*}{ Reduction osteotomy } & Positive & $11(57.9)$ & $7(63.6)$ & \multirow{2}{*}{0.53} \\
\hline & Negative & $8(42.1)$ & $4(36.4)$ & \\
\hline \multirow{2}{*}{ Pie-crusting } & Positive & $8(42.1)$ & $2(18.2)$ & \multirow{2}{*}{0.17} \\
\hline & Negative & $11(57.9)$ & $9(81.8)$ & \\
\hline
\end{tabular}

PS: Posterior Stabilized; CCK: Constrained Condylar Knee

Orthopedic Science

optimal preoperative planning of TKA, which is of paramount importance [10, 14].

In this study, we aimed at investigating factors that could be used in the identification of patients who might require a $\mathrm{CCK}$ prosthesis. Based on the results of the present study, the preoperative correctability of the deformity could be used as a critical factor in the prediction of the patients who might require a CCK prosthesis.
A significant association was also found between the preoperative correctability and the step of release so that a higher step of release was required in non-correctable deformities. The step of release was also significantly associated with the type of prosthesis. Thus, non-correctability results in the higher stages of release and more implication of CCK prosthesis.

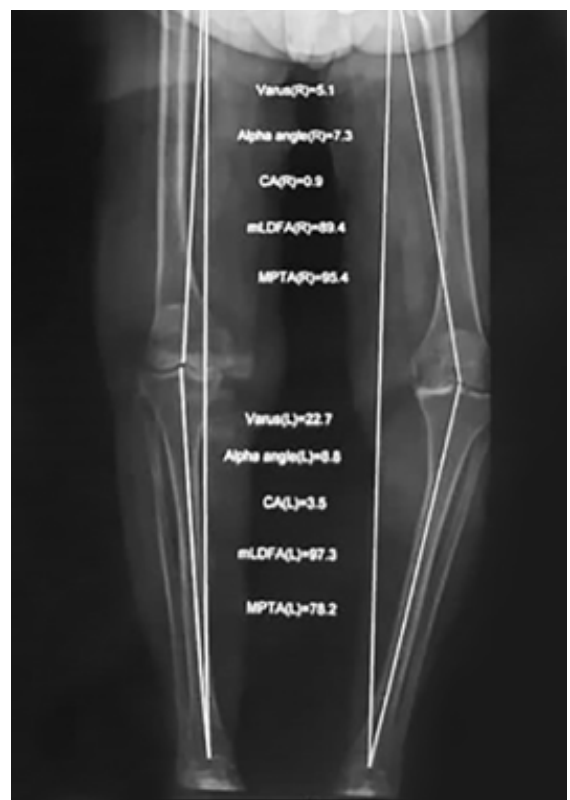

Orthopedic Science

Figure 1. Anteroposterior standing radiograph showing the severe genu varum deformity (varus angle $\geq 20^{\circ}$ ) 
Puah et al. compared the clinical and functional outcomes of CCK and PS in 38 paired patients who underwent TKA. There was no significant difference in 6-month knee extension, knee flexion, Oxford Knee Score, and 36-item short form survey total hip arthroplasty scores of the two study groups. There was no significant difference in 2-year knee extension, knee flexion, Oxford Knee Score, and SF-36 scores of the two study groups, as well. They concluded that using CCK in primary THA provides similar clinical and functional outcomes as those of PS prostheses, despite increased constraint [9]. The study of Rai et al. revealed the same results. However, they mentioned that using $\mathrm{CCK}$ has its complications [7]. Besides, CCK is associated with high cost, complexity, and bone removal. This evidence supports the importance of preoperative identification of patients who might need a CCK prosthesis in TKA.

Baldini et al. reviewed patients' or deformity-associated factors that can make TKA a challenging procedure. These factors include several previous operations and incisions, severe coronal deformities, a stiff knee, genu recurvatum, extra-articular deformities such as tibia vara, previous osteotomy around the knee, and chronic dislocation of the patella [15]. The present study revealed that the preoperative non-correctability of the deformity could group the factors, as pointed out in the study of Baldini et al.

Goltzer et al. aimed at determining if preoperative radiographic criteria of valgus knees can help to predict the extent of required soft tissue release, as well as the level of constraint needed to balance the knee. A total of 807 consecutive TKA standing hip-knee-ankle radiographs were analyzed in this study. Their analyses revealed that preoperative radiographic characteristics of the valgus knee could be implicated in predicting the extent of the lateral soft-tissue release and the necessity of constrained articulation in TKA. They suggested that this information could be useful to offer accurate preoperative counseling to patients and to ensure the availability of appropriate prosthesis during the operation [16].

The results of the present study revealed that preoperative clinical correctability could also be used as a factor to predict the amount of soft-tissue release, as well as the necessity of constrained articulation.

Some surgeons consider factors such as older age and gender in their decision-making to use a constrained articulation [16]. The results of the present study revealed no association between the demographic factors of the patients and the choice of prosthesis.
This study has some limitations. The main limitation of the study was the retrospective nature of the survey, as well as the small number of samples. Besides, the level of correctability was not determined quantitatively. We believe that the quantitative evaluation of preoperative correctability will result in the further codification of patients, who might require a CCK prosthesis. Therefore, we suggest further prospective investigations with larger sample sizes and quantitative evaluation of preoperative clinical correctability.

\section{Conclusion}

The preoperative clinical evaluation of correctability could be used in the identification of patients who may need a CCK prosthesis. This information could be used to facilitate surgical planning and efficiency by ensuring that appropriate prosthesis options are available when required. Furthermore, it may facilitate preoperative patients' counseling to address their expectations more accurately.

\section{Ethical Considerations}

\section{Compliance with ethical guidelines}

Written informed consent was obtained from patients to use their medical data.

Funding

This research did not receive any specific grant from funding agencies in the public, commercial, or not-forprofit sectors.

\section{Authors' contributions}

Conceptualization: Abolfazl Bagherifard; Study supervision: Mahmoud Jabalameli; Critical revision of the manuscript: Mohammad Rahbar; Data collection: Omid Shahpari; Interpretation of results and drafting the manuscript: Hossein Hajitaghi.

\section{Conflict of interest}

The authors declared no conflict of interests.

\section{References}

[1] Bagherifard A, Kadijani AA, Yahyazadeh H, Rezazadeh J, Mirkazemi M, Mirzaei A. Clinical and radiographic symptoms are strongly associated in males with knee osteoar- 
thritis, but not in females. Shafa Ortho J. 2018; 5(2):e68801. [DOI:10.5812/soj.68801]

[2] Tehrani-Banihashemi A, Davatchi F, Jamshidi AR, Faezi T, Paragomi P, Barghamdi M. Prevalence of osteoarthritis in rural areas of Iran: A WHO-ILAR COPCORD study. Int J rheumatic Dis. 2014; 17(4):384-8. [DOI:10.1111/1756185X.12312] [PMID]

[3] Mathiessen A, Conaghan PG. Synovitis in osteoarthritis: Current understanding with therapeutic implications. Arthritis research \& therapy. 2017; 19:18. [DOI:10.1186/ s13075-017-1229-9] [PMID] [PMCID]

[4] Thomas E, Peat G, Croft P. Defining and mapping the person with osteoarthritis for population studies and public health. Rheumatolo. 2014; 53(2):338-45. [DOI:10.1093/ rheumatology/ket346] [PMID] [PMCID]

[5] Kremers HM, Larson DR, Crowson CS, Kremers WK, Washington RE, Steiner CA, et al. Prevalence of total hip and knee replacement in the United States. J Bone Joint Surg American Vol. 2015; 97(17):1386-97. [DOI:10.2106/ JBJS.N.01141] [PMID] [PMCID]

[6] Canovas F, Dagneaux L. Quality of life after total knee arthroplasty. Ortho Traumatol. 2018; 104(1):S41-6. [DOI:10.1016/j.otsr.2017.04.017] [PMID]

[7] Rai S, Liu X, Feng X, Rai B, Tamang N, Wang J, et al. Primary total knee arthroplasty using constrained condylar knee design for severe deformity and stiffness of knee secondary to post-traumatic arthritis. J Ortho Surg Res. 2018; 13:67. [DOI:10.1186/s13018-018-0761-x] [PMID] [PMCID]

[8] Lachiewicz PF, Soileau ES. Ten-year survival and clinical results of constrained components in primary total knee arthroplasty. J Arthroplasty. 2006; 21(6):803-8. [DOI:10.1016/j.arth.2005.09.008] [PMID]

[9] Puah KL, Chong HC, Foo LSS, Lo NN, Yeo SJ. Clinical and functional outcomes: Primary constrained condylar knee arthroplasty compared with posterior stabilized knee arthroplasty. J Am Acad Orthop Surg Glob Res Rev. 2018; 2(2):e084. [DOI:10.5435/JAAOSGlobal-D-17-00084] [PMID] [PMCID]

[10] Tanzer M, Makhdom AM. Preoperative planning in primary total knee arthroplasty. J Am Acad Orthop Surg. 2016; 24(4):220-30. [DOI:10.5435/JAAOS-D-14-00332] [PMID]

[11] Lee SY, Yang JH, Lee YI, Yoon JR. A novel medial soft tissue release method for varus deformity during total knee arthroplasty: Femoral origin release of the medial collateral ligament. Knee Surg Relat Res. 2016; 28(2):153-60. [DOI:10.5792/ksrr.2016.28.2.153] [PMID] [PMCID]

[12] Bagheri F, Gharehdaghi M, Izanloo A, Rahimi H, Mirkazemi M. [Proximal tibial osteotomy in patients with varus knee deformity using MW technique: Evaluation of results (Persian)]. Shafa Ortho J. 2017; 4(3). [DOI:10.5812/ soj.11547]

[13] Bagherifard A, Jabalameli M, Mirzaei A, Khodabandeh A, Abedi M, Yahyazadeh H. Retaining the medial collateral ligament in high tibial medial open-wedge osteotomy mostly results in post-operative intra-articular gap reduction. Knee Surgery, Sports Traumatology, Arthroscopy. 2019:1-6. [DOI:10.1007/s00167-019-05473-8] [PMID]
[14] H E. Total knee arthroplasty for complex osteoarthritic varus knee deformities. Ortho \& Rheum Open Access J. 2018; 13(1):555851. [DOI:10.19080/OROAJ.2018.13.555851]

[15] Baldini A, Castellani L, Traverso F, Balatri A, Balato G, Franceschini $\mathrm{V}$. The difficult primary total knee arthroplasty: A review. Bone Joint J. 2015; 97(Suppl. 10):30-9. [DOI:10.1302/0301-620X.97B10.36920] [PMID]

[16] Goltzer O, Mroz TP, Temkit Mh, Clarke HD, Spangehl MJ. Preoperative radiographic valgus alignment predicts the extent of lateral soft tissue release and need for constraint in valgus total knee arthroplasty. Arthroplast Today. 2017; 3(4):303-8. [DOI:10.1016/j.artd.2017.06.003] [PMID] [PMCID] 